\title{
ALGUNOS ELEMENTOS CLAVE PARA MEJORAR LA CONVIVENCIA DESDE UN ENFOQUE COMUNITARIO KEY ELEMENTS FOR IMPROVING THE COMIMUNITY'S ABILITY TO LIVE TOGETHER IN HARMONY
}

\author{
Carina Hernández Leal (1) Carlos Sánchez Santos (1) \\ (1) Coordinadores del Servicio de Dinamización Vecinal del Ayuntamiento de Madrid
}

Resumen: En julio de 2011 se cumplían siete años desde el comienzo del Servicio de Dinamización Vecinal (SDV). Un dispositivo público que tiene como objetivo contribuir al logro de una convivencia armoniosa y enriquecedora entre todos los vecinos y vecinas de los barrios donde está presente, independientemente de su lugar de origen, cultura, religión, ideología o condición sexual. En todos estos años se han llevado a cabo cientos de acciones, con la participación de miles de personas, en el marco de la dinamización y la mediación comunitarias. Acciones desarrolladas a partir de un intenso trabajo en red, que han permitido generar importantes procesos de cambios individuales y colectivos.

Tras esta experiencia acumulada, hemos creído oportuno compartir, a modo de reflexión, algunos de los elementos clave que entendemos han guiado nuestra intervención hasta ahora. Pretendemos con ello hacer una aportación a ese proceso de aprendizaje continuo tan necesario para quienes, en tanto que profesionales, voluntariado, entidades privadas o administraciones públicas, tenemos como reto el contribuir a alcanzar una sociedad rica y diversa, desde un enfoque intercultural.

Palabras Clave: Interculturalidad, Ciudadanía activa, Sostenibilidad, Adaptabilidad, Servicio de Dinamización Vecinal.

Abstract: In July 2011 the Community Dynamics Service (Servicio de Dinamización Vecinal) of Madrid entered its seventh year of activity. The purpose of this public service is to help achieve harmonious and enriching coexistence among all members of the communities in which the service is present, regardless of their place of origin, culture, religion, ideology or sexual orientation. Over the years hundreds of activities have been organized, with the participation of thousands of people, in the framework of community dynamization and mediation. These actions are the product of hard work by the partners and they have generated significant change at the individual and collective levels.

Having accumulated such valuable experience, we believe it worthwhile for us to share our reflections on some of the key elements that have guided our intervention thus far. In doing so we hope to contribute to the process of ongoing learning of everyone who is involved, whether as a professional, a volunteer, a private entity or a public body, in the challenge of achieving a rich and inclusive society, based on an intercultural approach.

Key Words: Interculturality, Active Citizenry, Sustainability, Adaptability, Community Dynamics Service.

| Recibido: 15.09.2011 | Revisado: 29.11.2011 | Aceptado: 02.12.2011 | Publicado: 01.05.2012 |

Correspondencia: Carina Hernández Leal. Coordinadora del Servicio de Dinamización Vecinal del Ayuntamiento de Madrid. Federación Regional de Asociaciones Vecinales de Madrid, C/ Bocángel 2, 28028 Madrid. Tlf.: 917252909. Fax: 91.72506 23. Email: fravm@aavvmadrid.org. Página web. www.aavvmadrid.org 


\section{PUNTO DE PARTIDA DE LA INTERVENCIÓN COMUNITARIA DEL SERVICIO DE DINAMIZACIÓN VECINAL (SDV)}

Como decíamos, el SDV ${ }^{1}$ es un dispositivo público que está integrado en el /l Plan Madrid de Convivencia Social e Intercultural. Un plan director a través del cual el Ayuntamiento de Madrid articula todas las políticas municipales y recursos de integración y de gestión de la diversidad, desde un enfoque intercultural. EI SDV busca favorecer la convivencia cotidiana de vecinos y vecinas a través de las herramientas que ofrecen la dinamización y mediación comunitarias, estando presente, en la actualidad, en un total de 20 barrios/zonas de nuestro municipio: Almenara y Bellas Vistas (Tetuán), Prosperidad (Chamartín), Poblado A y B de Fuencarral (Fuencarral-El Pardo), Lavapiés y Embajadores (Centro), Almendrales y San Fermín (Usera), Los Rosales, Los Ángeles, San Andrés y San Cristóbal (Villaverde), San Isidro, Comillas y Alto de San Isidro (Carabanchel), Lucero (Latina), Valdebernardo (Vicálvaro), Quintana (Ciudad Lineal), Triángulo del Agua (Puente de Vallecas) y PAU Vallecas (Villa de Vallecas).

Uno de los elementos diferenciales que caracteriza al SDV y que refleja una de sus fortalezas tiene que ver con su presencia en las asociaciones vecinales. EI SDV trabaja con y desde estas entidades o, como solemos decir, "codo con codo" y por la mejora de la convivencia del barrio o territorio objeto de actuación.

No existe, que sepamos, iniciativa similar en nuestro país donde el acuerdo entre administración local y entidades ciudadanas haya dado como fruto la creación de una figura profesional, en el ámbito de la intervención comunitaria, que suponga una colaboración tan coordinada, constante y tan "a pie de calle". En ese sentido, creemos que el SDV sigue siendo una iniciativa de colaboración pionera entre lo público y lo privado, con grandes dosis de innovación. Este trabajo conjunto está contribuyendo, a nuestro entender, a aumentar el impacto de las políticas públicas en la sociedad, generando mayor grado de corresponsabilidad entre la ciudadanía y, por tanto, mayores cuotas de participación ciudadana, así como procesos de cambio mucho más sostenibles, como veremos posteriormente.

EI SDV toma como punto de partida, en la actualidad, una serie de conceptos y enfoques fruto, en algunos casos, de su evolución y madurez. Conceptos que definen su marco y modelo de intervención y que recogemos a continuación:

- El SDV entiende la interculturalidad como la interacción de las personas en una sociedad diversa y multicultural, pero dentro de un marco de igualdad y justicia. Aspiramos así a una sociedad donde esta interacción se lleve a cabo de manera respetuosa y donde las diferencias sean una oportunidad para el enriquecimiento, en lugar de una fuente de deterioro social y enfrentamiento inútil. Nos oponemos, por tanto, a concepciones, como la defendida por Huntington, a partir de su publicación El choque de Civilizaciones ${ }^{2}$ y creemos firmemente que la aspiración de nuestra sociedad debe seguir siendo la interculturalidad y el mestizaje, independientemente del contexto político o la coyuntura económica.

\footnotetext{
1 El SDV inicia su andadura en el año 2004 y en la actualidad depende de la Dirección General de Igualdad (Ayto. Madrid), estando gestionado por la Federación Regional de Asociaciones Vecinales de Madrid.

2 En su famoso libro, Huntington defiende la tesis de que, en el siglo XXI, los principales actores políticos serán las civilizaciones y que los conflictos de la política global no serán tanto ideológicos como conflictos entre civilizaciones (naciones y grupos nacionales).
} 
- Lograr una sociedad plenamente intercultural exige un esfuerzo bidireccional. Por ello, en su tarea, el SDV considera clave trabajar no solo con las personas de origen extranjero, sino también con la sociedad de acogida, desde la consideración de que la buena convivencia exige un esfuerzo y compromiso de todas. Para ello, el SDV articula acciones con todos los colectivos y franjas de edad, ya sean "autóctonos", o de origen extranjero. Este trabajo con unos grupos u otros viene definido, en última instancia, por las necesidades detectadas a través del diagnóstico que el SDV elabora de manera participada en cada territorio donde interviene.

- El barrio como una oportunidad para el encuentro, el intercambio y el enriquecimiento mutuo. La "vecindad", entendida como categoría sociológica, comparte no solo un territorio común, sino también una serie de acontecimientos sociales y retos que, en muchos casos, requieren la articulación de respuestas colectivas. Ser "vecino/a" es, por tanto, una categoría inclusiva, un "lugar común" que brinda la oportunidad de generar identidades y procesos compartidos, relegando a un segundo plano otras categorías basadas en elementos diferenciales, tales como el lugar de origen de cada cual, el sexo, la condición social, etc. El "barrio" y la "vecindad" son, por tanto, espacios privilegiados que el SDV toma como ámbito de actuación para el desarrollo de su misión.

- La convivencia intercultural entendida desde un enfoque integral y amplio. La promoción de la buena convivencia no solo tiene que ver con la sensibilización y el trabajo en valores, sino con un planteamiento mucho más amplio. A través de dicho planteamiento el SDV articula estrategias que buscan incidir en aquellos otros elementos y niveles que intervienen en la integración y la inclusión social de las personas de origen extranjero y los/as vecinos en general. Estas estrategias tienen que ver con el acceso efectivo de vecinos y vecinas a los recursos y ayudas públicas, con el ejercicio real de los derechos sociales y ciudadanos, así como el cumplimiento de los diversos niveles de corresponsabilidad, la promoción de procesos de desarrollo comunitario, el fortalecimiento de las redes sociales y asociativas, etc. Todas estas líneas conciben la convivencia como un proceso complejo y multidimensional, que exige un abordaje a múltiples niveles, evitando así intervenciones aisladas y parciales.

- La intervención en los diversos escenarios de lo comunitario. Lo comunitario incluye diversos espacios físicos: los espacios privados de las comunidades de vecinos; los espacios públicos, parques y jardines; los centros educativos... Además de estos escenarios, la intervención del SDV exige mantener una mirada atenta hacia otros espacios: los espacios organizacionales (aquellos en los que profesionales, recursos y entidades articulan iniciativas con el objeto de llevar a cabo una intervención conjunta y en red) y los espacios relacionales, allí donde están presentes e interactúan los grupos informales y la vecindad en general.

- Los dos niveles de intervención: 1) lo comunitario, desde un enfoque preventivo y 2) la mediación en conflictos. El Servicio de Dinamización Vecinal ha ido evolucionando desde sus comienzos. Este avance ha justificado un cambio en la denominación del servicio, cuyo nombre en sus orígenes era el de Servicio de Mediación Vecinal. Un cambio que no es casual, pues representa una evolución clara en el modelo de intervención, donde cada vez adquiere un mayor peso el enfoque preventivo, que podemos asociar más a la "dinamización vecinal", frente a la gestión y resolución de conflictos, vinculado a la "mediación vecinal". Así, se ha pasado de gestionar y abordar conflictos ya existentes, a trabajar preventivamente, evitando así acontecimientos sociales que pudieran suponer un deterioro de la convivencia 
comunitaria. No obstante, en este trabajo preventivo se mantienen las pautas y criterios que ofrece la metodología de mediación comunitaria (importancia de la corresponsabilidad y la participación de todos los agentes, necesidad de contar con interlocutores válidos...) para integrarlos en todos los procesos de intervención que se llevan a cabo.

- Participación y ciudadanía activa. La participación está inserta en todos los procesos que el SDV dinamiza e impulsa. Si en sus orígenes el SDV prima una intervención basada en el binomio "convivencia-conflicto", progresivamente se va incorporando el concepto de "participación" como estrategia trasversal e indisolublemente ligada a la promoción de la convivencia desde un enfoque preventivo y comunitario. Esta participación se articula y refleja a diferentes niveles: 1) cada barrio donde el SDV interviene cuenta con un plan de trabajo que se diseña, implementa y evalúa conjuntamente con la asociación vecinal de referencia y teniendo en cuenta los discursos y posicionamientos de otros agentes; 2) cualquier proceso, actuación o taller que se articula tiene en consideración las necesidades, demandas y opiniones comunes expresadas por los participantes del proceso (el 68\% de los talleres iniciados durante 2010 se hicieron a partir de una demanda explícita de la ciudadanía); 3) el profesional del SDV adopta un papel de dinamizador y facilitador de procesos, mientras que el protagonismo de estos lo tienen vecinos y vecinas, a quienes se anima a que sean participantes activos o sujetos (y no objetos) de sus propios procesos de cambio; 4) en la gestión de cualquier conflicto de convivencia, son las partes quienes aportan las soluciones y deciden cuándo el conflicto se considera resuelto.

- Los procesos de gestión y resolución de conflictos en los que interviene el SDV toman como referencia metodológica los principios de la mediación comunitaria. Según esta, el conflicto es consustancial a la vida misma y a las relaciones en sociedad. En sí mismo, un conflicto no es ni bueno ni malo. Solo es buena o mala la forma en que lo gestionemos. Desde cierta mirada, los conflictos pueden ser incluso provechosos para el progreso de una sociedad, si somos capaces de afrontarlos de manera adecuada. Se trata así de buscar soluciones donde ambas partes resulten ganadoras. Algo particularmente necesario en el ámbito comunitario, el de la vecindad, donde las partes de un conflicto van a tener que seguir manteniendo algún tipo de interacción, bien porque son vecinos, bien porque acuden a un centro educativo, bien porque disfrutan de un espacio público común, etc. En la base de la mediación está el favorecer, por tanto, la Cultura del Pacto, dando paso al diálogo para alcanzar acuerdos.

Dicho esto, recogemos a continuación las cuatro grandes líneas de actuación a través de las cuales el SDV articula su intervención en los territorios donde está presente:

- LÍNEA 1. Sensibilización intercultural y lucha contra el racismo y la xenofobia, a través de la creación y promoción de espacios de encuentro e intercambio, la formación y sensibilización en valores cívicos y de interculturalidad, la revalorización de la cultura popular y representativa de la diversidad multicultural, así como la promoción y recuperación de la memoria histórica del barrio.

- LÍNEA 2. Promoción de la convivencia y la participación social, mediante la dinamización de Procesos de Desarrollo Comunitario, la formación de grupos activos juveniles, la sensibilización y empoderamiento de grupos de mujeres y la inclusión e integración social a través del aprendizaje del castellano. 
- LÍNEA 3. Gestión y mediación en conflictos, a partir de la normalización y dinamización del uso de los espacios públicos, la formación y sensibilización en torno a la Cultura del Pacto y los valores de la mediación, así como la gestión de conflictos en diversos ámbitos de lo comunitario.

- LIINEA 4. Información y orientación, a partir de atenciones individuales y grupales, así como la organización de actos informativos, charlas, mesas a pie de calle y el intercambio permanente de información con otros agentes.

Cada una de estas líneas se ha venido desarrollando en los diferentes barrios en mayor o menor grado, adaptándose al diagnóstico social de cada territorio y concretándose en la realización de cientos de talleres y acciones permanentes, así como de eventos y encuentros puntuales, tales como ${ }^{3}$ : Año Nuevo Chino, Taller de bailes urbanos, Taller de documental, Día contra el Racismo y la Intolerancia, Historia e Historias de Tetuán, Noches de Ramadán, El Rapeadero de Lavapiés, Liga de Baloncesto Callejero, Procesos de Desarrollo Comunitario en Alto de San Isidro, Triángulo del Agua y PAU Vallecas, Jornadas Encuentros y Desencuentros en los Espacios Públicos, Taller de Mediación Educativa TKA, Revive la Reve, Fiestas de San Isidro...

Para hacernos una idea de la dimensión que supone la actividad del SDV, sirvan algunos datos referidos a los indicadores de evaluación durante 2010. Año en el cual se llevaron a cabo 2086 atenciones individualizadas, gracias en parte a las 3350 colaboraciones con profesionales, con los que se trabajó conjuntamente en un total de 118 mesas y espacios de coordinación barrial y distrital. Por otra parte, el SDV promovió o colaboró, a lo largo de todo el año, en 289 actividades comunitarias, en las que participaron un total de 26610 personas, mientras que el número de conflictos de convivencia gestionados fue de 94 , siendo resueltos satisfactoriamente un $27,6 \%$ de ellos.

Datos que nos hacen pensar que el SDV ha contribuido a que, pese al contexto actual de crisis económica, Madrid siga siendo una ciudad abierta y acogedora, en la que no se han producido, en los últimos años, brotes destacables de racismo social y xenofobia.

\section{VALORES Y ELEMENTOS CLAVE DEL SDV}

Hasta ahora hemos trazado el marco del servicio, fruto, como decíamos, de un aprendizaje y evolución permanentes. En este apartado daremos un paso más allá, para centrarnos no tanto en el qué hacemos sino en el cómo lo hacemos. Repasaremos aquí aquellos valores y elementos clave que, como comentábamos al inicio, están enraizados en la metodología y las dinámicas de trabajo que el SDV ha venido desarrollando estos últimos años. Valores que se concretan en cinco y que, adecuadamente enfocados, creemos que pueden ayudar a generar un impacto mayor en aquellas actuaciones (y no solo las del SDV) que buscan generar procesos de cambio individuales y colectivos, desde un ámbito de intervención comunitaria. Estos valores son:

- Cercanía y respuesta inmediata. Uno de los elementos diferenciales clave para que el SDV lleve a cabo con éxito su misión es la cercanía al barrio y sus redes sociales. Cercanía fruto

3 Toda la previsión de actividades del SDV se puede consultar mensualmente en www.madrid.es 
del trabajo desde y con las asociaciones vecinales, así como en coordinación con buena parte de los recursos y servicios públicos, pero también privados, presentes en el territorio. El SDV es, probablemente, en estos momentos, uno de los recursos a disposición de las administraciones públicas más cercano y en contacto con la ciudadanía y sus necesidades. Esta cercanía, unida a la confianza que se genera, permite que la canalización de dichas necesidades sea lo más ágil posible, detectándolas de manera prácticamente simultánea a los cambios sociales que se van produciendo.

- La cercanía con el territorio lleva a un segundo aspecto o valor que caracteriza de manera muy notable al Servicio de Dinamización Vecinal. Este valor se podría expresar como la capacidad para ofrecer una respuesta rápida ante aquellos acontecimientos en la vida y la convivencia del barrio que lo requieran. Acontecimientos que, en muchos casos, no entienden del "vuelva usted mañana" o la cita para dentro de una semana.

- Adaptabilidad y cambio permanente. EI SDV cuenta con una alta capacidad para dar una respuesta adecuada a los entornos cambiantes y novedades que se generen. Cada territorio donde el SDV interviene cuenta con una realidad social específica. Para desarrollar su intervención, el profesional elabora un análisis de manera continua y participada, en colaboración con otros agentes del territorio, entre los que destaca la asociación vecinal como informante clave. Diagnóstico que se va enriqueciendo en el día a día y que exige una alta capacidad de adaptabilidad del/la dinamizador/a, de cara a dar respuestas coherentes a la especificidad propia del territorio en el que interviene, prestando especial atención a las personas de origen extranjero.

- Con y desde las asociaciones vecinales. La presencia en las asociaciones vecinales y el trabajo "codo con codo" permite que los objetivos del SDV sean apropiados por estas. Para ello existe una coordinación diaria a través de la cual se diseñan, implementan y evalúan conjuntamente los procesos. Ello permite que los procesos sean mucho más sostenibles, que es el reto de cualquier iniciativa planteada desde el SDV. De hecho, durante 2010, el 62\% de las actividades del SDV contaron con la participación o la colaboración directa de la asociación vecinal. Esto está siendo particularmente importante en un contexto de crisis económica y de merma de recursos, donde muchas de las acciones a día de hoy no serían posibles sin la aportación (económica, material, humana...) de dichas entidades. Un resultado claro de esto ha sido la posibilidad de desarrollar actividades permanentes o talleres en los locales y sedes de las asociaciones (el 64\% de los talleres durante 2010 se han celebrado en estas).

La presencia en las asociaciones vecinales proporciona al SDV, además, otro tipo de recursos intangibles: su alto grado de conocimiento del entorno, su capacidad para articular respuestas a las necesidades detectadas, generando sinergias, su capacidad de representatividad de los vecinos, canalizando sus discursos hacia enfoques propositivos y constructivos, etc.

- Trabajo en red. EI SDV ha demostrado una alta capacidad para liderar procesos que tienen detrás un importante trabajo en red, es decir: en colaboración con otros recursos, entidades e instituciones. De hecho, casi un 29\% de las actividades comunitarias del SDV se han articulado, durante 2010, en el marco de una mesa o espacio de trabajo en red. Además, son 118 el número de mesas y espacios de coordinación donde ha estado presente el SDV durante este año. Espacios donde, a través del enfoque comunitario, se logra incorporar la visión de vecinos y vecinas, tan necesaria para una administración moderna que aspira a ser más eficaz y eficiente en sus políticas públicas, además de que cuenten cada día con mayor representatividad. La 
creación e impulso de este tipo de espacios para la articulación del trabajo en red hace, además, más sostenible y, sobre todo, eficiente la intervención. Permite, además abordar determinadas problemáticas desde un enfoque integral. Pero no solo eso: el impulso del trabajo en red está contribuyendo a dar un auténtico salto en los modelos de intervención social de las administraciones. La ciudadanía (y en especial la ciudadanía menos informada, que suele coincidir con aquellos colectivos más desprotegidos), demanda cada vez más de las administraciones la lógica de funcionamiento de "ventanilla única". No podemos esperar a que esta ciudadanía conozca todos los resortes y mecanismos de nuestras administraciones, sino que debemos ofrecérselos de la manera más cercana y accesible, canalizando sus demandas de la forma más adecuada. En lo local, una Administración Inteligente, en el contexto actual, es aquella que, aun contando con unas competencias concretas y delimitadas, se muestra preocupada por todas las problemáticas de tipo social que tengan sus ciudadanos, tratando de darles respuesta, o bien, de canalizar lo más adecuadamente posible dicha respuesta. En ese sentido, desde el SDV estamos contribuyendo a alcanzar este reto gracias, en buena parte, a la cercanía del profesional con las personas y su territorio.

- Sostenibilidad de procesos. Todos los procesos que el SDV impulsa en el ámbito comunitario tienen como reto la sostenibilidad, es decir: la necesidad de que dichos procesos sean apropiados por los agentes que ya están presentes en el territorio y, en especial, por la ciudadanía. Esta sostenibilidad solo es posible cuando los procesos que se abren parten de la demanda real y las necesidades de vecinos y vecinas. En este sentido, el/la profesional se encarga de operativizar y dinamizar estos procesos, pero en ningún caso decide qué camino han de seguir. Existen ejemplos claros que muestran de manera nítida como los objetivos y los retos del SDV se van incorporando dentro de las agendas de las asociaciones vecinales, así como dentro de otras entidades, grupos informales y vecinos en general. Se da, por tanto, una evolución que trasforma los sujetos receptores de los servicios, en sujetos activos de los procesos.

\section{CONCLUSIONES}

A modo de conclusión, son varios los elementos clave que nos ha parecido importante destacar. Probablemente, no estén todos los que son, pero sí son todos los que están. Algunos de ellos definen el marco de la intervención, así como conceptos básicos de partida del Servicio de Dinamización Vecinal, tales como la idea de interculturalidad, la de ciudadanía activa, etc. Otros elementos clave hacen referencia a valores del servicio, que impregnan la metodología y las técnicas de intervención.

En coherencia con esto, desde el SDV consideramos que es fundamental, en el marco de una intervención comunitaria que busca promover la buena convivencia, no solo alcanzar los objetivos planteados, en términos de impacto en la sociedad sino, además, hacerlo de una determinada manera y, en nuestro caso, a través de los valores que hemos repasado: cercanía y respuesta inmediata, adaptabilidad y cambio permanente, con y desde las asociaciones vecinales, a través de un importante trabajo en red y teniendo en cuenta la sostenibilidad de los procesos.

En consecuencia, evaluar una intervención comunitaria exige mirar más allá de los resultados más inmediatos y tangibles de las acciones. Indicadores tales como la cantidad de participantes o el número 
de acciones desarrolladas que, en muchos casos, son tomados como base para evaluar recursos y dispositivos públicos, no son suficientes para garantizar si el impacto en la sociedad es el adecuado y el que esta necesita. Por tanto, los valores de un servicio, recurso o intervención, están íntimamente ligados a la calidad de dicha intervención o viceversa: hablar de la calidad de una intervención social y comunitaria remite directamente a una serie de valores y elementos clave que deben ser también evaluados en esta. Si no tenemos conciencia de nuestros valores y elementos clave y, por tanto, no evaluamos en coherencia con estos, estamos, muy probablemente, condenados a hacer "activismo por activismo", sin que seamos capaces de llevar a cabo una evaluación adecuada, herramienta esencial para introducir procesos de aprendizaje continuos en la intervención. Procesos de aprendizaje que, como decíamos al principio, nos ayudan a crecer y a continuar dando respuesta a los retos que la sociedad nos va presentando.

\section{AGRADECIMIENTOS}

El presente artículo es fruto de la sistematización y evaluación del Servicio de Dinamización Vecinal, llevada a cabo durante 2011. Tarea que no hubiera sido posible sin la intensa labor de compañeros y compañeras como Laura Martínez Díaz, Marcela Manubens, Ernesto Esteso, María Gálvez Dávila y Javier Santos Bueno. Vaya por delante nuestro agradecimiento y reconocimiento a su esfuerzo, compromiso e ilusión permanentes.

\section{BIBLIOGRAFÍA}

Huntington, S.P. (1997): El choque de civilizaciones y la reconfiguración del orden mundial. Barcelona: Editorial Paidós. 